\title{
Cáncer pulmonar en adultos jóvenes no fumadores Reporte de casos
}

\section{Lung cancer in nonsmoking young adults Report of two cases}

Luis Gerardo García-Herreros( ${ }^{(1)}$; Adolfo Torres Galán ${ }^{(2)}$; Diana Ale-jandra Holguín Sanabria(2); Julio César Granada ${ }^{(3)}$

\begin{abstract}
RESUMEN
El cáncer de pulmón es una entidad que afecta a la población mundial, teniendo una alta tasa de mortalidad comparado con otros tipos de cáncer. Cuando un cáncer se presenta se requiere la ocurrencia de mutaciones genéticas para que la célula proge-nitora se torne neoplásica, por tanto, la mayoría de cánceres ocurren después de la cuarta década de la vida; en el cáncer de pulmón se cumple este enunciado siendo muy poco frecuente en personas menores de 40 años. Hay algunas características que son diferentes en jóvenes incluyendo una alta incidencia de adenocarcinoma y una menor tasa de presentación hombre-mujer (1).

En los estudios realizados se ha comprobado una relación directa del tabaquismo con este tipo de cáncer, pero no se pueden olvidar otros factores de riesgo propuestos en la literatura mundial, entre ellos están la infección por TBC, vivir en países industriali-zados y la quema de aceites.

En el presente reporte de caso se presentan dos casos, dos pacientes de género fe-menino y sin aparentes factores de riesgo a quienes se les diagnosticó cáncer de pul-món. Fueron llevadas a procedimientos quirúrgicos y actualmente se encuentran en tratamiento con quimioterapia.
\end{abstract}

Palabras clave: cáncer de pulmón, adultos jóvenes, adenocarcinoma, tabaquismo.

\section{ABSTRACT}

Lung cancer is a worldwide disease whose mortality rate is high with respect to other types of cancer. In cases of cancer, genetic mutations must occur so that the progenitor cell becomes neoplastic. Consequently, most cancers occur after the fourth decade of life. This stands for lung cancer, which is very uncommon in people aged less than 40 years. Some characteristics are peculiar of younger cases, including a high incidence of adenocarcinoma and a lower male/female ratio (1).

Studies have shown a direct relationship of tobacco smoking with this type of cancer. However, other risk factors described in the world literature, such as TBC infection, living in industrialized countries, and burning of oils, should not be forgotten.

Two cases are described in this report. Both patients are females without apparent risk factors diagnosed with lung cancer. They were intervened surgically and are currently undergoing chemotherapy.

Key words: lung cancer, young adults, adenocarcinoma, tobacco.

Rev Colomb Neumol 2011; 23(1): 26-29.

(1) MD., Cirujano de Tórax, Jefe Departamento de Cirugía, Fundación Santa Fe de Bogotá. Bogotá, Colombia.

(2) MD., Interno vocacional Cirugía de Tórax, Fundación Santa Fe de Bogotá. Bogotá, Colombia.

(3) MD., Cirujano de Tórax, Trasplante Pulmonar, Fundación Santa Fe de Bogotá. Bogotá, Colombia.

Correspondencia: Doctor Luis Gerardo Garcíaherreros, correo electrónico: Iggarciaherreros@gmail.com y Doctor Adolfo Torres Galán, correo electrónico: adolfotg@gmail.com

Recibido: 9 de febrero de 2011. Aceptado: 15 de febrero de 2011. 


\section{INTRODUCCIÓN}

El cáncer primario de pulmón, es una entidad con una alta tasa de mortalidad con res-pecto al cáncer de otros órganos (2). Ocurre en la mayoría de casos, en personas en-tre 50-75 años de edad y solamente un $3 \%-5 \%$ se presenta en adultos menores de 40 años (3). La literatura mundial muestra que el tipo de cáncer de pulmón más común es el adenocarcinoma (4). Diferentes factores de riesgo se han propuesto a lo largo de los años; principalmente el tabaquismo $85 \%$, seguido de infección por TBC y quema de aceites.

El inicio temprano de la enfermedad se ha atribuido a fumadores pesados, como se documenta en más del 90\% de pacientes jóvenes con cáncer en países occidentales; sin embargo, se debe recordar que en Asia, Latinoamérica y Turquía sólo se reporta este antecedente en cerca de $40 \%$ a $50 \%$ de pacientes menores de 40 años con cán-cer de pulmón (1). La relación del tabaquismo con este cáncer es tan estrecha, que un estudio realizado por la Sociedad de Cirujanos Torácicos de Estados Unidos concluye que el riesgo de muerte y complicaciones pulmonares después de la resección quirúr-gica aumenta si el paciente fuma en el periodo perioperatorio y disminuye si este hábi-to cesa (14).

Estudios en países de Asia han evidenciado que las presentaciones atípicas son más frecuentes en mujeres (4). Si bien la literatura mundial reporta mayoría de casos de presentaciones atípicas en mujeres, en nuestro país es inusual esta forma de presen-tación. Adicionalmente, la sobrevida de estos pacientes es corta $y$, por lo general, se presentan con estadios muy avanzados de la enfermedad, en los cuales no es posible ofrecer un tratamiento curativo.

Factores de riesgo para pronóstico se han propuesto: pérdida de peso, tipo histológi-co, estado actual del paciente al momento del diagnóstico, sin que estos datos sean totalmente conclusivos. Los casos en pacientes jóvenes se presentan con el subtipo histológico de carcinomas de pulmón, van desde $46 \%$ del adenocarcinoma, seguidos con $14 \%$ para el de célula pequeña, $12 \%$ células escamosas, $8 \%$ células grandes indi-ferenciadas y otros $20 \%$ (6). Paris y colaboradores publicaron en 2010 un estudio en el que no se encontró asociación entre la aparición de adenocarcinoma con la edad (5).

En el mundo se han reportado casos de presentaciones atípicas de cáncer pulmonar: en niños (7), mujeres en embarazo (8). Como se ha mencionado, el cáncer de pulmón en jóvenes es una entidad poco frecuente, sin embargo, los reportes documentan la alta tasa de mortalidad cuando ocurre $(9,10)$. Según el estudio rea- lizado en el año 2000, por el hospital de Harvard Medical School, el doctor David Sugarbaker y el doc-tor Skarin y colaboradores (6) en un estudio con 91 pacientes menores de 40 años; la tasa de supervivencia en años, según el estadio, varía desde 9,75 para estadios I y II, 2,56 para IIIA, 0,83 para IIIB, 0,70 estadios IV. En este mismo estudio se concluye que en los pacientes jóvenes que presentan esta entidad, no se encontró un tipo histológi-co biológicamente más agresivo que en los adultos mayores, sin embargo, al momen-to de su diagnóstico la enfermedad esta claramente más avanzada, lo cual empeora su pronóstico.

En este reporte de caso se describen dos pacientes de género femenino de 30 y 36 años de edad, que fueron llevadas a cirugía y se les diagnosticó adenocarcinoma de pulmón, sin tener tabaquismo como factor de riesgo. En el momento se encuentran en tratamiento con quimioterapia, posterior a su procedimiento quirúrgico.

\section{CASO 1}

Se trata de una paciente de 30 años de edad quien consulta por cuadro clínico de 1 mes de evolución de astenia, adinamia, disnea leve.

La paciente consultó en varias ocasiones a centros hospitalarios por los síntomas refe-ridos. La radiografias del torax (Figuras 1 y 2) muestran una masa que compromete el parenquima pulmonar, en el lóbulo inferior izquierdo. La tomografia axial computariza-da evidencia una masa de consistencia sólida, con densidad de tejidos blandos, que compromete la lingula y en su mayoría el lóbulo inferior izquierdo, sin compromiso de pleura, no derrame pleural, ganglios mediastinales. Se decidió programar para cirugía para resección de la masa: lobectomía inferior total por toracotomía izquierda. No se realizó biopsia por broncoscopia dado que no cambiaría en nada la conducta. Durante el procedimiento se observó compromiso del lóbulo superior, por lo que se decide realizar una neumonectomía, más vaciamiento ganglionar. Se realiza toma de biopsias por congelación intraoperatorias que reportan adenocarcinoma de pulmón, mediana-mente diferenciado. Se finaliza el procedimiento sin complicaciones y se traslada a la paciente a UCI, dejando el tubo de tórax sin succión. En el cuarto día posoperatorio se decide dar de alta a la paciente y control por consulta externa. El reporte final de pato-logía fue un adenocarcinoma de pulmón moderadamente diferenciado, de tipo muco-productor, primario de pulmón, sin invasión vascular y linfática. Tejido pulmonar de otras áreas con inflamación granulomatosa crónica necrotizante y hialinización. Pleura visceral comprometida con tumor, ganglios linfáticos con enfermedad granulomatosa. 


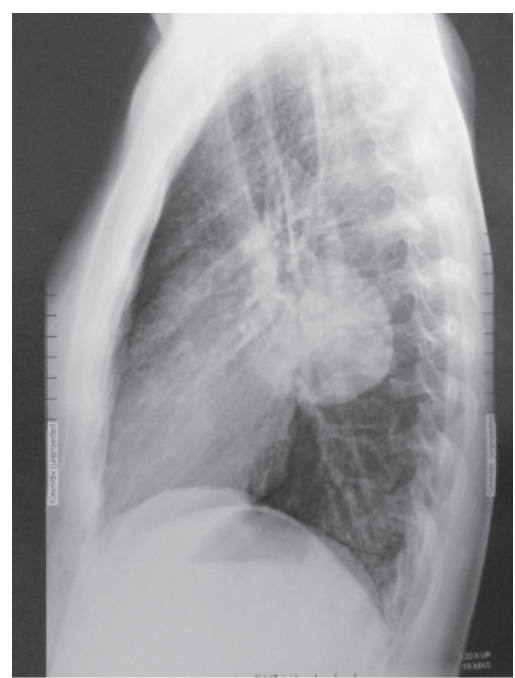

Figura 1. Proyección lateral. Se evidencia una masa de aspecto sólido de aproximadamente $5 \mathrm{~cm}$ de diámetro, que compromete el lobulo inferior izquier-do, segmento apicoposterior.

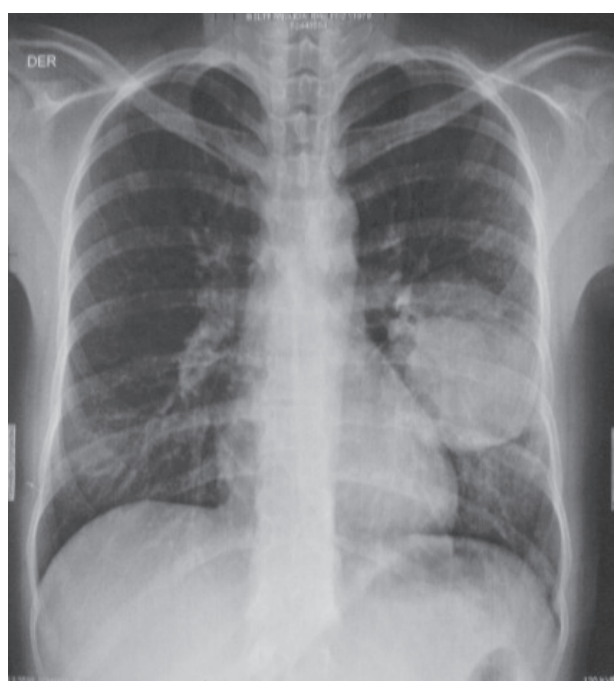

Figura 2. Proyección posteroaterior.

\section{CASO 2}

Paciente de 36 años de edad, no fumadora, quien consulta a su ortopedista por cua-dro clínico de 3 meses de evolución de dolor en hombro derecho y región cervical. Se realizaron diferentes estudios imaginológicos y terapia física. Dentro de los estudios, solicitaron una tomografia axial computarizada y una resonancia magnética de colum-na, donde se observan lesiones que comprometen el pulmón. Es valorada por neumo-logía quienes solicitan nuevamente una tomografia axial del tórax (Figuras 3 y 4), la cual confirmo la presencia de derrame pleural, pero adicionalmente evidenció múltiples nódulos pulmonares de menos de $5 \mathrm{~mm}$. Se le realizó una fibrobroncoscopia donde se tomaron 4 biopsias del lóbulo medio en las que se encontró lesión. El reporte de patología fue tumor maligno de origen desconocido. Se le solicita PET CT y gamagrafía ósea. En las imágenes del PET CT se observa un área de hipercaptación a nivel de la pleura apical y un claro aumento del derrame pleural (Figura 5) por lo que se decide realizar una toracentesis y un ultrasonido del tiroides.

Estudio histoquímico positivo para malignidad, se interconsulta a cirugía de tórax para intervención quirúrgica. Es valorada y se programa para drenaje de derrame pleural, decorticación, pleurodesis y biopsia pulmonar por toracoscopia. Durante el procedi-miento quirúrgico se encontraron los siguientes hallazgos: compromiso de la pleura en su totalidad, incluyendo compromiso del paquete vasculonervioso, músculos de la pa-red del tórax, diafragma. El reporte definitivo de patología del

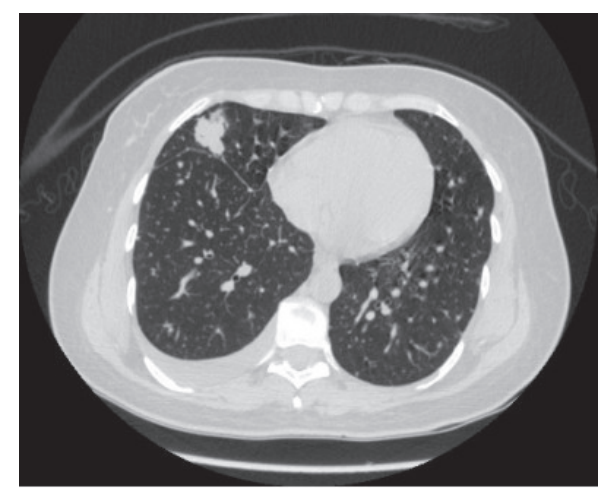

Figura 3. Tomografia axial computarizada, corte axial, donde se evidencia una gran lesión que compromete el hemitorax derecho y múltiples nódulos pulmonares que comprometen la totalidad del pulmón.

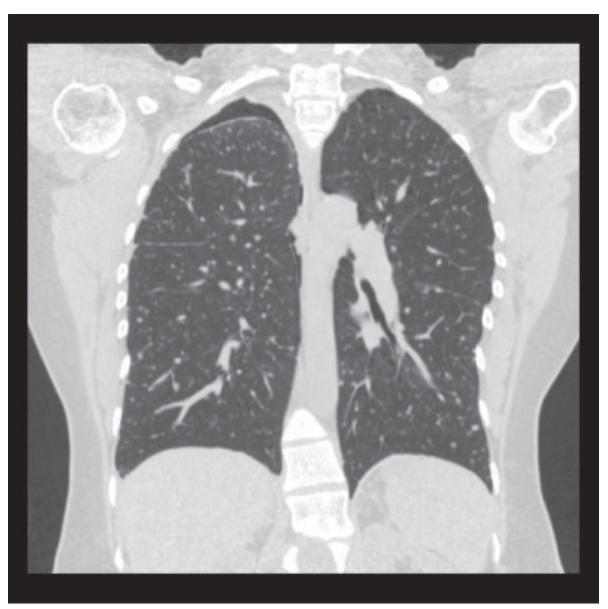

Figura 4. Tomografia computarizada corte longitudinal, donde se evidencia neumotó-rax y múltiples lesiones nodulares a lo largo de todo el parenquima pulmonar. 
citológico adenocarci-noma bien diferenciadoTTF1, citorekatina 7 con reactividad; ausencia de reactividad para citokeratina 20 y tiroglobulina.TAC de cuello: glándula tiroides con nódulo sólido, de ecogenicidad heterogenea en el tercio medio de 15,4 x 8.3 x 5,6 con pequeños fo-cos ecogénicos en el interior. Gamagrafía ósea negativa para metastasis. Biopsias por toracoscopia con reporte definitivo de adenocarcinoma moderadamente diferenciado mucoproductor probablemente de origen pulmonar. Hemorragia alveolar reciente y atelectasia, antracosis.

Pleura parietal: adenocarcinoma moderadamente diferenciado, fibrosis pulmonar, hiperplasia mesotelial difusa reactiva. Estudio de mutaciones EGFR negativo.

Actualmente, la paciente se encuentra en tratamiento con quimioterapia.

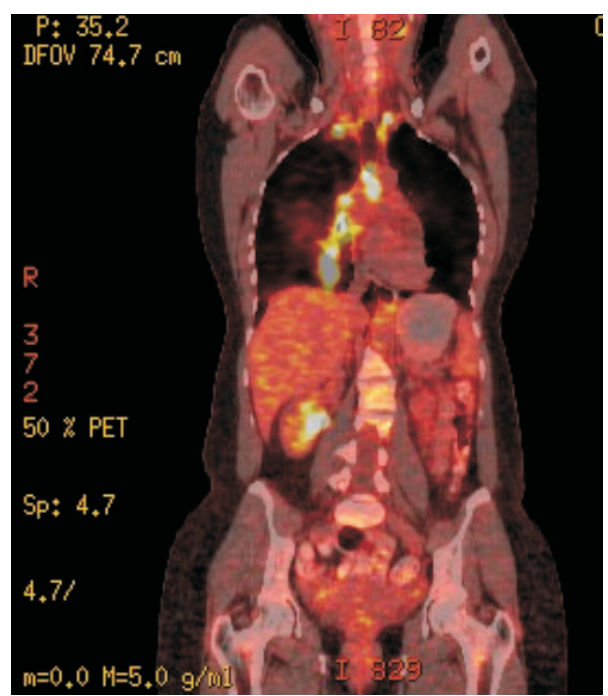

Figura 5. Tomografía por emisión de positrones. Zonas de hipercaptacion ápices y compromiso pleural difuso.

\section{DISCUSIÓN}

Si bien la incidencia del cáncer de pulmón no es despreciable, según estudios realiza-dos por la Universidad Nacional de Colombia; se estima que es más común en hom-bres 70/100.000 habitantes, en el este de Europa, frente a un 20/100.000 en países no industrializados como Colombia y la incidencia en mujeres se estima que es la mitad que en los hombres. En su mayoría se presenta en pacientes mayores de 50 años de edad y con factores de riesgo como el tabaquismo, que tienen una relación directa-mente proporcional. En la literatura mundial se han descrito casos donde el tabaquis-mo no es el factor de riesgo desencadenante $(2,6,7,8)$. Al igual que en uno de nues-tros casos, el reporte dado por
Park, en uno de sus pacientes presentaba infección por TBC, sin embargo, nuestra paciente no recibió de antemano tratamiento para ésta, debido a que no se conocía el diagnóstico de la infección y no tenía factores de riesgo para la misma.

Así como en el resto del mundo, donde la incidencia de presentaciones atípicas es mayor en mujeres, nuestros casos se presentan en mujeres donde no hay factor de riesgo aparente o conocido y el estadio en el que se diagnostica la enfermedad, ya es avanzado y de pronóstico reservado $(9,10)$. Si bien el cáncer de pulmón es un capitu-lo en el cual se escribe casi a diario, hay áreas de este que no se comprenden; por este motivo es necesario que nuevos casos se reporten y nuevos estudios se realicen para así poder conocer mejor esta enfermedad, ya que es una de las mayores causan-tes de muertes en el mundo.

\section{REFERENCIAS}

1. Cakir, et al. Basaloid squamous cell carcinoma of the lung: a rare tumor with a rare presentation. Lung Cancer 2007; 57: 109-111.

2. Daniel Silva Gontijo Penha. Bronchogenic carcinoma of squamous cells in a young pregnant woman. Annals of Diagnostic Pathology 2006; 10235-238

3. Greenlee RT, Murray T, Bolden S, et al. Cancer statistics. Ca-A Cancer J Clin 2000; 50: 7-33.

4. Jeong-A Park. Adenocarcinoma of lung in never smoked children. Lung Cancer 2008; 61:266-9.

5. Kuo C, Chao J, et al. Non-small Cell Lung Cancer in Very Young and Very Old Patients. Chest 2000; 117; 354-357

6. Liu NS, Spitz MR, Kemp BL, Cooksley C, Fossella FV, Lee JS, et al. Adenocar-cinoma of the lung in young patients: the M.D. Anderson experience. Cancer 2000; 88: 1837-41.

7. Mason D, Subramanian S, et al, Impact of Smoking Cessation Before Resection of Lung Cancer: A Society of Thoracic Surgeons General Tho-racic Surgery Database Study, Ann Thorac Surg 2009; 88: 362-71.

8. Murthy S, Reznik S, et al. Winning the Battle, Losing the War: The Noncu-rative "Curative" Resection for Stage I Adenocarcinoma of the Lung. Ann Thorac Surg 2010; 90: 1067-74.

9. Nesbitt J, Putnam J. Survival in Early-Stage Non-Smll Cell Lung Cancer. Ann Thorac Surg 1995; 60: 466-72.

10. Paris $C$, et al. Relationships between lung adenocarcinoma and gender, age, smoking and occupational risk factors: A casecase study. Lung Cancer 2010; 68: 146-53.

11. Ramalingam S, Pawlish K, Gadgeel S, Demers R, Kalemkerian GP. Lung can-cer in young patients: analysis of a surveillance, epidemiology, and end results database. J Clin Oncol 1998; 16: 651-7.

12. Sekine I et al. Young lung cancer patients in Japan: Different characteristics between the sexes. Ann Thorac Surg 1999; 67: 1451-5.

13. Sekine I, Nishiwaki $Y$, et al. Young Lung Cancer Patients in Japan: Dif-ferent characteristics between the sexes. Ann Thorac Surg S 1999; 67: 1451-5.

14. Skarin A, et al. Lung cancer under age 40. Lung Cancer 32 (2001) 255-264 